\title{
Towards LPRM-based soil moisture retrievals with multi-angular microwave observations from SMOS
}

\author{
S. Liu ${ }^{\text {a }}$, C.-H. Su ${ }^{\text {a, D. Ryu }}{ }^{\text {a }}$ and K. Kim ${ }^{\text {a }}$ \\ ${ }^{a}$ Department of Infrastructure Engineering, The University of Melbourne, Parkville, Victoria, Australia \\ Email: shucil@student.unimelb.edu.au
}

\begin{abstract}
Passive microwave remote sensing by the Soil Moisture and Ocean Salinity (SMOS) satellite enables observations of surface soil moisture at global scale. This involves a retrieval process that uses a radiative transfer model to relate satellite-observed radiances to geophysical variables such as soil dielectric constant and soil moisture. The L-MEB (L-band Microwave Emission of the Biosphere) based retrieval products of SMOS have previously been evaluated to show reasonably good agreement with ground measurements. An alternative product is based on the Land Parameter Retrieval Model (LPRM), which has been adapted for a wide range of $\mathrm{X}$ - and $\mathrm{C}$-band sensors to retrieve soil moisture from single-angle observations. From the implementation viewpoint, LPRM is attractive in its limited requirements for additional auxiliary parameters and in its simple geophysical parameterization. The research gaps lie in the limited previous study on retrieving SMOS multi-angular observations using LPRM. The development of a LRPM-retrieved SMOS product is also motivated by establishing an algorithmic consistency across multiple C-, X- and L-band sensors, allowing them to be compared more directly for relative performance.

This study considers the LPRM-like retrieval algorithm based on generic Radiative Transfer Equation (RTE) and uses a controlled numerical experiment to determine the properties of LPRM when acting on synthetic multi-angular SMOS observations. The influences of observational uncertainties, model parameter uncertainties and multi-angle observations (c.f. single-angle) are evaluated on the retrieval performance. In particular, the Markov Chain Monte Carlo (MCMC) algorithm is used to quantify the uncertainties in, and correlations between, retrieved parameters, under these influences.
\end{abstract}

The main findings of this study are listed as follows,

- According to retrieval sensitivity analysis, vegetation optical depth $(\tau)$ and surface temperature $\left(T_{s}\right)$ are more critical parameters in the retrieval model compared to surface roughness and scattering albedo, and they may be retrieved simultaneously with soil moisture in a 3-parameter retrieval configuration;

- The uncertainty in brightness temperature $\left(T_{B}\right)$ has more significant impact on a vegetated-wet scenario than on a bare-dry case: vegetated-wet surface can tolerate $2 \mathrm{~K}$ brightness temperature uncertainty to achieve the target retrieval accuracy whereas bare-dry surface can tolerate as much as $8 \mathrm{~K} T_{B}$ uncertainty;

- MCMC results demonstrate the advantages of LPRM soil moisture retrieval with multi-angular $T_{B}$ observations over single-angle retrievals in terms of higher robustness and less uncertainty in retrieval results.

This work therefore provides guidance to adapting LPRM for SMOS data and soil moisture retrieval at continental scale.

Keywords: $\quad$ Microwave remote sensing, soil moisture retrieval, Soil Moisture and Ocean Salinity (SMOS), Land Parameter Retrieval Model (LPRM), sensitivity analysis 
Liu et al., Towards LPRM-based soil moisture retrievals with multi-angular microwave observations from SMOS

\section{INTRODUCTION}

Microwave satellite sensors allow estimation of land-surface soil moisture minimally interrupted by clouds and vegetation cover. These satellite-retrieved soil moisture products have shown potential in improving (hydrologic, land-surface and weather) model analyses and/or prediction, studying global change in water cycle and drought monitoring (e.g. Brocca et al., 2012). Several studies have argued that passive remote sensing at L-band (1.1-1.7 GHz) is one of the most promising techniques due to low sensitivity to cloud and vegetation contamination, and high sensitivity to soil moisture fluctuations at deeper depths. These have led to the ESA's Soil Moisture and Ocean Salinity (SMOS) mission, as well as the NASA's Soil Moisture Active and Passive (SMAP) mission. SMOS is unique in terms of its instrumental design - its Microwave Imaging Radiometer by Aperture Synthesis (MIRAS) makes radiance observations of a single land surface scene at multiple incidence angles. The official ESA algorithm, L-MEB (L-band Microwave Emission of the Biosphere) retrieval method, is sophisticated by accounting for spatial heterogeneity in a mixed scene, dependency between surface roughness and soil moisture, and soil temperature difference between surface and subsurface sampling depth in the presence of different soil types. Many auxiliary data are required for its implementation. An alternative algorithm is the Land Parameter Retrieval Model (LPRM), which has been applied to single-angle observations from a wide range of $\mathrm{C}$ - and $\mathrm{X}$-band sensors, namely SMMR, TMI, AMSR-E and WindSat. LPRM has certain implementation advantages due to its lesser dependency on auxiliary data and simplified parameterization of land surface characteristics (e.g. de Jeu et al., 2009). Evaluation studies comparing the L-MEB based SMOS product with LPRM-based AMSR-E product found that their performances are comparable (e.g. Su et al., 2013).

It is therefore of particular interest to apply LPRM to SMOS data. Specifically, it is desirable to maintain an algorithmic consistency across historical and present passive sensors, especially for the purpose of combining them to construct multi-decadal soil moisture climatic record within the ESA's Climate Change Initiative (CCI) program. The LPRM products are part of the passive and active-passive ESA CCI soil moisture product (e.g. Wagner et al., 2012). Indeed, the application of LPRM to SMOS has been achieved recently by Van der Schalie et al. (2015) and was tested in the Murrumbidgee catchment, southeast Australia. The LPRM algorithm was applied to observations at three different incidence angles $\left(45,52.5\right.$ and $\left.60^{\circ}\right)$ separately to yield soil moisture estimates that are similar to the L-MEB estimates. The retrieved values at different angles are statistically identical within the confidence intervals. However, inversion of a retrieval model to fit single-angle observations can be expected to be more susceptible to observational errors than fitting to multiangle data. Multi-angle data can provide tighter constraints on the model parameter values and retrieved values, although the amount of soil moisture information diminishes with decreasing incidence angles.

Within this context, we pursue an adaption of LPRM algorithm for SMOS multi-angle observations. To achieve this, this paper investigates the following research questions. (1) What are the model parameters that can be estimated simultaneously with soil moisture through inversion of radiative transfer equation (RTE) model with simple parameterization? (2) What is the maximum level of observational errors to achieve a benchmark target of $0.04 \mathrm{~m}^{3} / \mathrm{m}^{3}$ retrieval errors? (3) What are the levels of improvement of using multi-angle data over single-angle data in the retrieval process? For these purposes, we use controlled numerical experiments based on a generic implementation of RTE with simple model parameterization akin to LPRM, to maximize clarity of our finding. We also use the Markov Chain Monte Carlo (MCMC) method to accurately determine the joint probability distribution amongst model parameters (including soil moisture), allowing unbiased estimation of their dependencies and uncertainties.

The paper is organized as follows. Sec. 2 reviews the RTE model in brief and describes our controlled experiments. Sec. 3 presents the results on retrieval sensitivity and Sec. 4 the MCMC analyses. Sec. 5 offers our concluding remarks.

\section{METHODOLOGY}

\subsection{Forward Model}

The radiative transfer principle of Mo et al. (1982), describing the upwelling radiative emission from land surface contributed by soil, canopy and atmosphere layers can express observed brightness temperature at satellite sensor $T_{B}$ with the following Radiative Transfer Equation (RTE),

$T_{B(P)}=e_{r(P)} T_{s} \Gamma_{v}+(1-\omega) T_{C}\left(1-\Gamma_{v}\right)+\left(1-e_{r(P)}\right)(1-\omega) T_{C}\left(1-\Gamma_{v}\right) \Gamma_{v}$,

where $P$ refers to vertical or horizontal polarization, $\Gamma_{v}$ the vegetation transmissivity, $\omega$ the single-scattering albedo, $e_{r(P)}$ the rough surface emissivity and $T_{s}$ the surface effective soil temperature, which is assumed to be 
Liu et al., Towards LPRM-based soil moisture retrievals with multi-angular microwave observations from SMOS

equal to $T_{c}$, the vegetation canopy temperature in LPRM. In other words, LPRM is more suited for periods when there is a thermal equilibrium between soil layer and vegetation.

The $T_{B}$ observations are subject to scattering and absorption by vegetation. In particular, $\Gamma_{\mathrm{v}}=\exp (-\tau / \cos u)$ in Eq. 1 is a function of the vegetation optical depth $\tau$ and incidence angle $u$. The surface roughness affects surface emissivity by increasing the scattering effect and reducing the sensitivity of emissivity to soil moisture variations. Accordingly, Choudhury et al. (1979) developed an empirical roughness correction model given by $e_{r(P)}=1-R_{S(P)} \exp \left(-h^{2} \cos ^{2} u\right)$, where $R_{s}$ is smooth-surface reflectivity, and $h$ is regarded as an effective roughness parameter. The Fresnel reflectivity equations allow us to relate $R_{s}$ to soil dielectric constant $\kappa$. In turn, empirically-derived dielectric mixing models with known information about the soil porosity, wilting point (or sand/clay fractions) and soil temperature, enable inference of volumetric soil moisture from the $\kappa$ estimate. The Wang-Schmugge model is chosen for this study.

To summarize, the observed brightness temperature can be expressed as a function of five unknown a priori model parameters $\left\{\kappa, T_{c}, \omega, \tau, h\right\}$ that must be resolved either from auxiliary data or from the retrieval (model inversion) process. The parameters $\left\{T_{c}, \omega, \tau, h\right\}$ can be regarded as auxiliary parameters. In LPRM, $\tau$ is retrieved simultaneously with $\kappa$, and $T_{c}$ is given by either $\mathrm{K}_{\mathrm{a}}$-band radiance observations or from models. Constants $\omega$ and $h$ are used for C- and X-band retrievals, although de Jeu et al. (2009) parameterized $h$ as a function of soil moisture and incidence angles for L-band retrieval with airborne data.

\subsection{Synthetic Experiment}

SMOS-like multi-angular $T_{B}$ is generated by forward model (1) according to each defined test scenario (Table 1), representing the actual land surface emission - 'referenced' $T_{B}$ at multiple incidence angles ranging from 0 to $60^{\circ}$ (at $1^{\circ}$ intervals) and at the two polarizations. A 'measured' $T_{B}$ is generated by adding Gaussian random errors to the 'referenced' $T_{B}$. The Gaussian parameter error structure is more preferable because the synthetic true $T_{B}$ ('measured' $T_{B}$ ) is more likely to have the similar feature with the real-life TB observed by satellite and the results are therefore more reliable and convincing. The 'measured' $T_{B}$ is input to the retrieval model to estimate soil dielectric constant $\kappa$ and soil moisture, amongst other model parameters. The retrieval process involves optimizing the values of $\kappa$ (and other prescribed model parameters) such that the model simulates $T_{B}$ values that most closely match 'measured' $T_{B}$. The optimization is set up as a nonlinear, least-square minimization, where the objective function is based on the difference between 'simulated' and 'measured' $T_{B}$. We consider different configurations of the retrieval process by choosing different auxiliary parameter(s) for simultaneous estimation with soil moisture.

\subsection{Scenario Definition}

Our synthetic experiments are based on six scenarios (listed in Table 1), distinguished by different soil moisture and vegetation canopy conditions. The roughness parameter $h$ is set constant to 0.2 , representing relatively smooth surface, which is consistent with the agricultural and natural areas surface roughness conditions (e.g. Panciera et al., 2009). The dependence of $h$ on soil moisture has been ignored for simplicity in this preliminary study. At L-band, $\omega$ is generally found in the literature to be low and for most algorithms it is assumed to be zero (e.g. Wigneron et al., 2007), which is similarly assumed in our study.

\section{THE RETRIEVAL SENSITIVITY ANALYSIS}

The known soil moisture and auxiliary parameter values are used as the synthetic truth value to simulate 'measured' SMOS $T_{B}$. The modelled $T_{B}$ is generated by forward model, with varying two parameters (soil moisture and one of auxiliary parameters) within the entire parameter feasible space: $\theta \in[0,0.7] \mathrm{m}^{3} / \mathrm{m}^{3}$, $\tau \in[0,1], \omega \in[0,0.3], h \in[0,1.5]$, and $T_{s} \in[273,323] K$. Root-mean-square error (RMSE) between measured and simulated $T_{B}$ over $0-60^{\circ}$ angles is computed, and plotted as a function of the two parameter values in Figure 1 for six scenarios. To allow direct comparisons between variables, the parameter values were normalized so that their feasible ranges match the 0-1 range ( 0 represents the lower limit and 1 represents the upper limit). For accurate single-parameter retrieval of soil moisture $\theta$ with a priori specification of the four auxiliary parameters, it is desirable for the global minimum of the RMSE surface to be well-defined along the $y$-axis ( $\theta$ axis) within a narrow trough, and global minimum along the $x$-axes to be poorly defined within a wide trough. In other words, the soil moisture can be accurately estimated even in the presence of inaccurate values of the auxiliary parameters; the retrieval is insensitive to the auxiliary parameters. By extension, for accurate two-parameter retrieval of soil moisture with one of the auxiliary parameters, the global minimum should be well-defined along both axes so that the retrieval is sensitive to the both parameters. 
Liu et al., Towards LPRM-based soil moisture retrievals with multi-angular microwave observations from SMOS

Table 1. Selected auxiliary parameter values for six scenarios.

\begin{tabular}{|c|c|c|c|c|c|c|c|}
\hline Scenario & $\theta\left(\mathrm{m}^{3} / \mathrm{m}^{3}\right)(\kappa)$ & $\tau$ & $\omega$ & $h$ & $T_{s}(\mathrm{~K})$ & Soil/Veq & on Characteristic \\
\hline 1 & $0.03(3.5)$ & \multirow{3}{*}{0.00} & \multirow{6}{*}{0} & \multirow{6}{*}{0.2} & \multirow{6}{*}{290} & Dry & \multirow{3}{*}{ Bare soil } \\
\hline 2 & $0.2(11.1)$ & & & & & Moist & \\
\hline 3 & $0.4(27)$ & & & & & Wet & \\
\hline 4 & $0.03(3.5)$ & \multirow{3}{*}{0.4} & & & & Dry & \multirow{3}{*}{ Vegetated soil } \\
\hline 5 & $0.2(11.1)$ & & & & & Moist & \\
\hline 6 & $0.4(27)$ & & & & & Wet & \\
\hline
\end{tabular}

With these criteria in mind, we compare the three bare soil cases with three vegetated soil cases in Figure 1. The presence of vegetation cover widens the troughs for all parameter combinations, which suggest greater susceptibility to retrieval errors. Increasing soil moisture appears to have the same effects. For the former, it is not surprising as the vegetation canopy attenuates the microwave emission of the underlying soil layer, effectively reducing the soil water information in the observations. For $\theta-h$ and $\theta-\omega$, troughs about the global minima generally have a longer extent along the $h$ and $\omega$ axes compared to $\tau$ and $T_{s}$ axes, showing lower sensitivity to $h$ and $\omega$ when soil moisture is retrieved. Specifically for $\omega$ under bare soil scenarios, the minimum along the $\omega$ axis does not exist because in the absence of vegetation, $\omega$ is non-determinate as per Equation 1 (the second and third terms containing $\omega$ are cancelled). The retrieval shows some sensitivity to $h$ in the bare wet soil and vegetated wet soil cases, and sensitivity to $\omega$ in the vegetated cases. The diagonalshaped trough suggests correlations in errors, i.e., biases in retrieved or input (as a priori) auxiliary parameter values will lead to biases in the retrieved soil moisture. The directions of the biases between $\theta$ and $h$ are the same, whereas they are opposite between $\theta$ and $\omega$.

The results show clear association between errors in $\theta$ and errors in $\tau$ and $T_{s}$; in other words, the retrieval errors in $\theta$ are sensitive to errors in these two auxiliary parameters. Furthermore, the sensitivity increases with increasing soil water content. The larger extents of the troughs along $\tau$ and $T_{s}$ axes indicate that small errors in $\tau$ and $T_{s}$ result in large errors in retrieved soil moisture. Therefore, $\tau$ and $T_{s}$ have the most significant impact on accuracy of retrieved $\theta$. Given these associations, it is conceivable to retrieve these critical auxiliary parameters jointly with soil moisture. In particular, this was exploited in LPRM to estimate $\theta$ and $\tau$ simultaneously. Henceforth, we focus on the retrieval of three parameters, namely $\theta, \tau$ and $T_{s}$.

\section{MARKOV CHAIN MONTE CARLO ANALYSIS}

MCMC is a stochastic sampling process for realistic statistical modelling. In this study, the DiffeRential Evolution Adaptive Metropolis (DREAM) algorithm developed by Vrugt (2015) is used. DREAM is a multichain MCMC simulation algorithm that automatically tunes the scale and orientation of the proposed (the prior) distribution of the retrieval model parameters to determine their posterior distributions that are conditioned on the $L_{2}$-based retrieval objective function. It is used here to investigate the influence of observational errors in input (multi-angle) $T_{B}$ observations and the benefits of using multi-angle observations over single-angle observations. Five iterative chains (10,000 iterations for each chain) for each parameter are proposed and overall 50,000 evaluations are conducted with the sum of square errors (SSE) objective function, which is used to form the likelihood function for MCMC exploration.

\subsection{Impacts of uncertainty in $T_{B}$}

We consider different levels of observational errors, with errors' standard deviation ranging from $0.5 \mathrm{~K}$ to $8 \mathrm{~K}$, for four particular scenarios (bare-dry, bare-wet, vegetated-dry and vegetated-wet in Table 1). The posterior distribution of the retrieved soil moisture and the two identified auxiliary parameters $\left(\tau\right.$ and $\left.T_{s}\right)$ are determined by the MCMC method. Specifically, the distribution of the Markov Chain ensemble after sufficiently many function evaluations (when the statistics of the ensemble become stationary) represents the distribution of the parameter values.

Figure 2 plots the RMSE in soil moisture and the ranges of retrieved parameters as a function of $T_{B}$ error standard deviations. In Figure 2(a), the dashed black line represents the target soil moisture accuracy of 0.04 $\mathrm{m}^{3} / \mathrm{m}^{3}$. The $T_{B}$ uncertainty has a more substantial impact on soil moisture retrieval under wet conditions, since the retrieved soil moisture RMSE is much higher than that of dry ones which can tolerate large $T_{B}$ uncertainty, as much as $8 \mathrm{~K}$. Meanwhile, as expected, the $\theta$ retrieval under vegetated soil scenarios is less accurate compared with bare soil condition, which is consistent with our finding from the sensitivity analysis where vegetated cover reduces soil emission information used for determining the $\theta$. 
Liu et al., Towards LPRM-based soil moisture retrievals with multi-angular microwave observations from SMOS
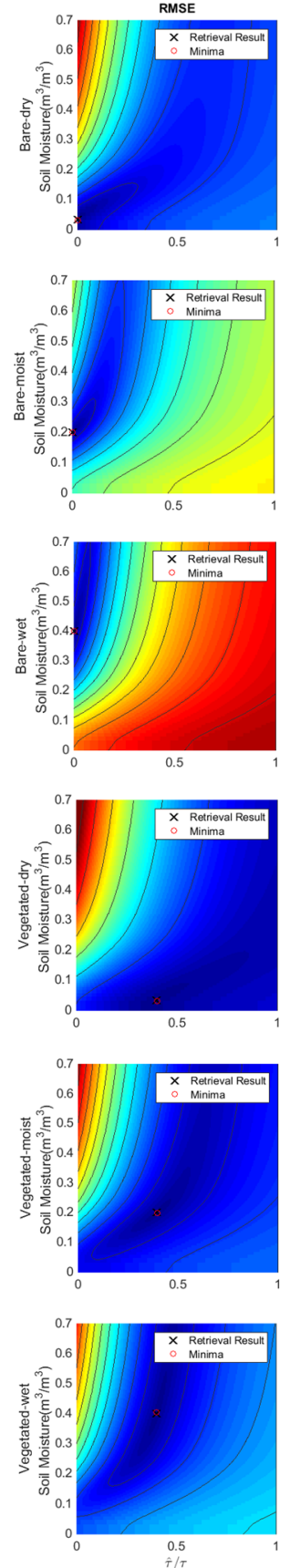
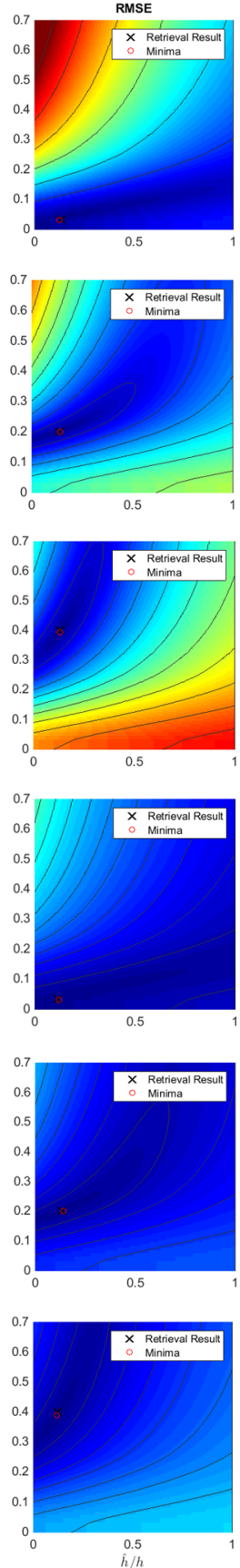
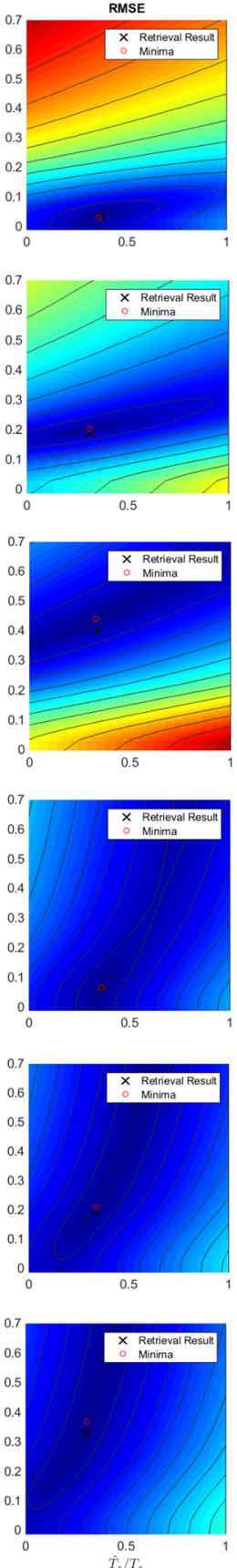
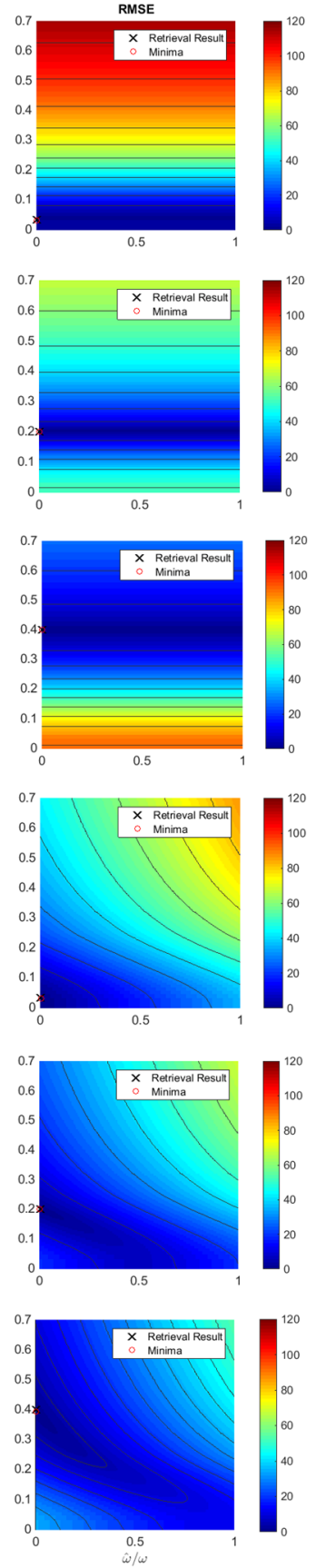

Figure 1. $T_{B}$ RMSE distribution for six scenarios of two-parameter combinations. RMSE is computed from the iterative difference between referenced and simulated $T_{B}$.

The three parameters ranges derived from MCMC chains are presented in Figure 2(b). $\kappa$ has been converted to soil moisture using the dielectric mixing model and the $y$-axis is normalized with respect to synthetic true value. Apparently $T_{s}$, indicated by the red boxes, is the most reliable retrieval result; however, retrieved $\theta$ is influenced significantly by $T_{B}$ uncertainty. This is partially because soil moisture has to be obtained using the dielectric mixing model, where both $\kappa$ and $T_{s}$ are model inputs. In other words, the uncertainties in these two parameters contribute to the soil moisture uncertainty.

Given that the instrumental radiometric accuracy of SMOS is $2-3 \mathrm{~K}$, this synthetic evaluation shows that retrievals over vegetated-wet soil are the most challenging given its lower tolerance to observational errors. While the results show that the 3-parameter retrieval has relative high robustness and applicability in other land surface conditions, reducing the number of parameters may be warranted to minimize the retrieval errors, especially over highly vegetated and high moisture environments. For instance, soil temperatures derived from numerical models and WindSat satellite (e.g. Parinussa et al., 2012), have been used for LPRM, 
Liu et al., Towards LPRM-based soil moisture retrievals with multi-angular microwave observations from SMOS

whereas L-MEB uses the soil temperature data from simplified modeling approach using two moisture and temperature data at land surface and at depth.

\subsection{Benefit of Multi-angular LPRM Retrieval}

The single-angular $T_{B}\left(45^{\circ}\right)$ and multiangular $\left(0-60^{\circ}\right)$ are tested under $1 \mathrm{~K} T_{B}$ uncertainty scenarios. Typically, LPRM is applied to high incidence angle observation, ranging from $45^{\circ}$ to $55^{\circ}$, therefore in MCMC study, the selection of single angle is $45^{\circ}$ (e.g. van der Schalie et al., 2015). The referenced $T_{B}$ is generated from the
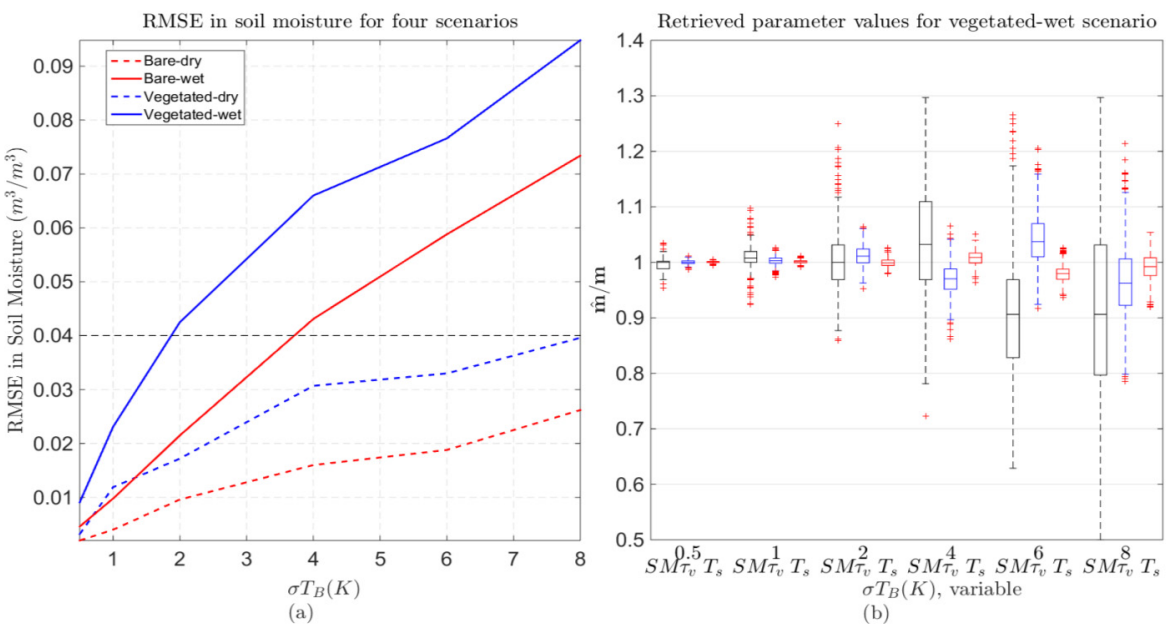

Figure 2. Statistics of the MCMCs under different level of errors in $T_{B}$. (a) RMSE in soil moisture for four scenarios; (b) Statistics of the retrieved parameter values (SM, $\tau_{v}, T_{s}$ ) for vegetated-wet scenario.

test parameter set: $S M=0.2 \mathrm{~m}^{3} / \mathrm{m}^{3}$ (i.e., $\kappa=11.1$ ), $\tau=0.25, \omega=0.03, h=0.2$ and $T_{s}=290 \mathrm{~K}$. After around 2,000 iterations, chains for multi-angular $T_{B}$ became converged, and chains for single-angular $T_{B}$ failed to converge after the entire iterations. By visually comparing the plotmatrix of the multi-chain behavior during stationary state for two types of $T_{B}$ input in Figure 3, the distribution of parameters can be obtained.

Parameter values for each plot matrix have been normalized (against the true values) and the diagonal histograms show the marginal distribution of a single parameter. The comparison of the parameter distributions between multi-angular and single-angular MCMC in Figure 3 shows the relative robustness between two retrieval approaches. The range of parameter value for multi-angular retrieval is centralized to the synthetic truth and follows a Gaussian distribution. The single-angular MCMC results demonstrate that parameters tend to spread across wider parameter space and are deviated from the truth. Further, the identified critical parameter $T_{s}$ and $\tau$ have narrower distribution compared with $h$ and $\omega$, demonstrating the need for retrieving $T_{s}$ and $\tau$ simultaneously with soil moisture or having accurate a priori values.

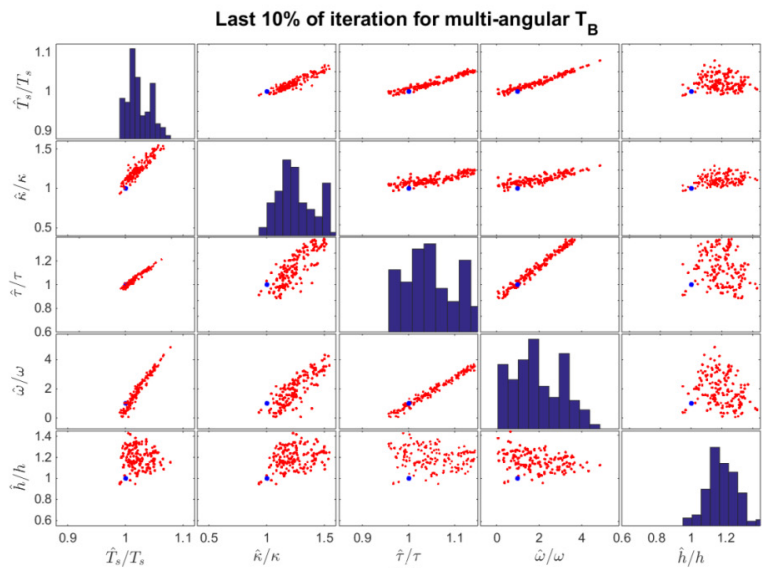

(a)

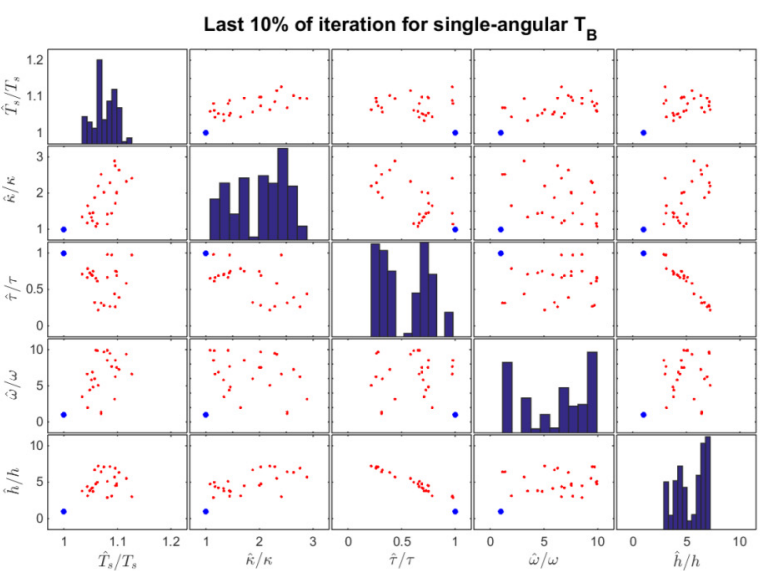

Figure 3. Plot matrix of last $10 \%$ iteration for multi- and single-angular (at $45^{\circ}$ ) $T_{B}$ with $1 \mathrm{~K}$ error. Note that the range of the $x$ and $y$-axes are different between (a) and (b); blue dots indicate the synthetic true values.

It is observed that the paired scatter plots in plot matrix for multi-angular $T_{B}$ indicate that $S M-T_{s}, S M-\tau, S M-$ $\omega, T_{s}-\tau, T_{s}-\omega$ and $\tau$ - $\omega$ are clearly correlated and these parameters are not mutually independent; whereas, for single-angular $T_{B}$ MCMC plotmatrix, there is less dependence demonstrated by all the parameters for singleangular MCMC results. The difference in MCMC results reveals that the multi-angular $T_{B}$ soil moisture retrieval has low uncertainty in retrieval parameters, validating the advantages of LPRM SMOS multi- 
Liu et al., Towards LPRM-based soil moisture retrievals with multi-angular microwave observations from SMOS

angular $T_{B}$ soil moisture retrieval over the single-angular retrieval, in terms of high robustness and less uncertainty in retrieved parameters.

\section{CONCLUSIONS}

Several conclusions can be drawn from the synthetic exploration of LPRM SMOS-like retrieval. First of all, the sensitivity analysis demonstrated that $\tau$ and $T_{s}$ are more critical parameters in the retrieval model. They can be retrieved simultaneously with soil moisture in a 3-parameter retrieval setup. Further, by testing the 3parameter retrieval under a range of $T_{B}$ uncertainties using MCMC method, the thresholds for observational error for four idealized water-vegetation scenarios are determined, and the uncertainty in $T_{B}$ has the least impact on bare-dry condition but has greater influence on vegetated-wet scenario. Lastly, the benefits of LPRM soil moisture retrieval using multi-angular $T_{B}$ observations over single-angle retrieval are demonstrated by MCMC analysis result. There is higher robustness and less uncertainty in retrieval results compared to single-angular LPRM. The future research opportunity could focus on field validation of LPRM against ground soil moisture measurement data and testing three-parameter retrievals against two-parameter retrievals. One such location is the Murrumbidgee catchment located in southeast Australia, where the dense soil moisture monitoring stations and various land cover types are available.

\section{ACKNOWLEDGMENTS}

This research was supported by The University of Melbourne, Melbourne School of Engineering's Early Career Researcher Grant.

\section{REFERENCES}

Brocca, L., Moramarco, T., Melone, F., Wagner, W., Hasenauer, S., \& Hahn, S. (2012). Assimilation of surface-and root-zone ASCAT soil moisture products into rainfall-runoff modeling. Geoscience and Remote Sensing, IEEE Transactions on, 50(7), 2542-2555.

Choudhury, B., Schmugge, T. J., Chang, A., \& Newton, R. (1979). Effect of surface roughness on the microwave emission from soils. Journal of Geophysical Research: Oceans (1978-2012), 84(C9), 5699-5706.

de Jeu, R. A., Holmes, T. R., Panciera, R., \& Walker, J. P. (2009). Parameterization of the land parameter retrieval model for L-Band observations using the NAFE'05 data set. Geoscience and Remote Sensing Letters, IEEE, 6(4), 630-634.

Mo, T., Choudhury, B., Schmugge, T., Wang, J., \& Jackson, T. (1982). A model for microwave emission from vegetation-covered fields. Journal of Geophysical Research: Oceans (1978-2012), 87(C13), 11229-11237.

Panciera, R., Walker, J. P., Kalma, J. D., Kim, E. J., Saleh, K., \& Wigneron, J.-P. (2009). Evaluation of the SMOS L-MEB passive microwave soil moisture retrieval algorithm. Remote Sensing of Environment, 113(2), 435-444.

Parinussa, R. M., Holmes, T. R., \& De Jeu, R. A. (2012). Soil moisture retrievals from the WindSat spaceborne polarimetric microwave radiometer. Geoscience and Remote Sensing, IEEE Transactions on, 50(7), 2683-2694.

Su, C.-H., Ryu, D., Young, R. I., Western, A. W., \& Wagner, W. (2013). Inter-comparison of microwave satellite soil moisture retrievals over the Murrumbidgee Basin, southeast Australia. Remote Sensing of Environment, 134, 1-11.

van der Schalie, R., Parinussa, R., Renzullo, L., van Dijk, A., Su, C.-H., \& de Jeu, R. (2015a). SMOS soil moisture retrievals using the land parameter retrieval model: Evaluation over the Murrumbidgee Catchment, southeast Australia. Remote Sensing of Environment, 163, 70-79.

Vrugt, J. A. (2015). Markov chain Monte Carlo Simulation Using the DREAM Software Package: Theory, Concepts, and MATLAB Implementation.

Wagner, W., Dorigo, W., de Jeu, R., Fernandez, D., Benveniste, J., Haas, E., \& Ertl, M. Fusion of active and passive microwave observations to create an essential climate variable data record on soil moisture. ISPRS Annals of the Photogrammetry, Remote Sensing and Spatial Information Sciences, XXII ISPRS Congress, I-7, 315-321.

Wigneron, J.-P., Kerr, Y., Waldteufel, P., Saleh, K., Escorihuela, M.-J., Richaume, P., Ferrazzoli, P., De Rosnay, P., Gurney, R., \& Calvet, J.-C. (2007). L-band Microwave Emission of the Biosphere (LMEB) Model: Description and calibration against experimental data sets over crop fields. Remote Sensing of Environment, 107(4), 639-655. 